The INL is a

U.S. Department of Energy

National Laboratory

operated by

Battelle Energy Alliance

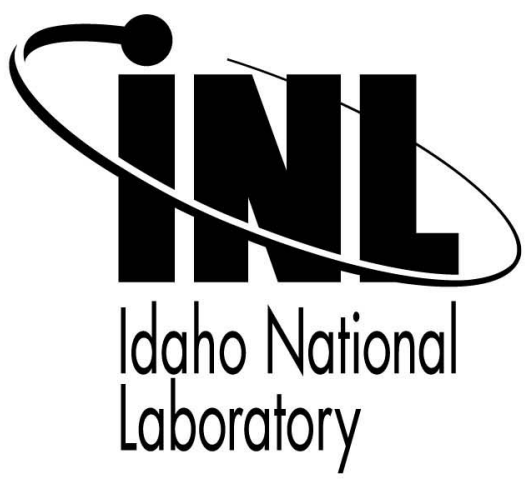

INL/CON-08-14427

PREPRINT

\section{Mining Process and} Product Information from Pressure Fluctuations Within a Fuel Particle Coater

\section{ASME $4^{\text {th }}$ International Topical Meeting on High Temperature Reactor Technology}

Douglas W. Marshall Charles M. Barnes

\author{
September 2008
}

This is a preprint of a paper intended for publication in a journal or proceedings. Since changes may be made before publication, this preprint should not be cited or reproduced without permission of the author. This document was prepared as an account of work sponsored by an agency of the United States Government. Neither the United States Government nor any agency thereof, or any of their employees, makes any warranty, expressed or implied, or assumes any legal liability or responsibility for any third party's use, or the results of such use, of any information, apparatus, product or process disclosed in this report, or represents that its use by such third party would not infringe privately owned rights. The views expressed in this paper are not necessarily those of the United States Government or the sponsoring agency. 


\section{MINING PROCESS AND PRODUCT INFORMATION FROM PRESSURE FLUCTUATIONS WITHIN A FUEL PARTICLE COATER}

\author{
Douglas W. Marshall \\ Battelle Energy Alliance, Idaho National Laboratory \\ Idaho Falls, ID, USA
}

\author{
Charles M. Barnes \\ Battelle Energy Alliance, Idaho National Laboratory \\ Idaho Falls, ID, USA
}

\section{ABSTRACT}

The Next Generation Nuclear Power/Advanced Gas Reactor (NGNP/AGR) Fuel Development and Qualification Program included the design, installation, and testing of a 6inch diameter nuclear fuel particle coater to demonstrate quality TRISO fuel production on a small industrial scale. Scale-up from the laboratory-scale coater faced challenges associated with an increase in the kernel charge mass, kernel diameter, and a redesign of the gas distributor to achieve adequate fluidization throughout the deposition of the four TRISO coating layers.

TRISO coatings are applied at very high temperatures in atmospheres of dense particulate clouds, corrosive gases, and hydrogen concentrations over $45 \%$ by volume. The severe environment, stringent product and process requirements, and the fragility of partially-formed coatings limit the insertion of probes or instruments into the coater vessel during operation.

Pressure instrumentation were installed on the gas inlet line and exhaust line of the 6-inch coater to monitor the bed differential pressure and internal pressure fluctuations emanating from the fuel bed as a result of bed and gas "bubble" movement. These instruments are external to the particle bed and provide a glimpse into the dynamics of fuel particle bed during the coating process and data that could be used to help ascertain the adequacy of fluidization and, potentially, the dominant fluidization regimes.

Pressure fluctuation and differential pressure data are not presently useful as process control instruments, but data suggest a link between the pressure signal structure and some measurable product attributes that could be exploited to get an early estimate of the attribute values.

\section{INTRODUCTION}

The second phase of the NGNP/AGR Fuel Development and Qualification Program included the design, installation, and testing of a 6-inch diameter nuclear fuel particle coater at a Babcock \& Wilcox Nuclear Operations Group facility in Lynchburg, VA, to demonstrate TRISO fuel production on an industrial scale. Scale-up faced the challenges associated with a 20-fold increase in the kernel charge to the coater (relative to the 2-inch, laboratory-scale coater used in process development), a $21 \%$ increase in the kernel diameter $(350 \mu \mathrm{m}$ to $425 \mu \mathrm{m}$ kernel), and the redesign of a gas distributor to achieve adequate fluidization throughout the deposition of the four TRISO coating layers shown in Figure 1.

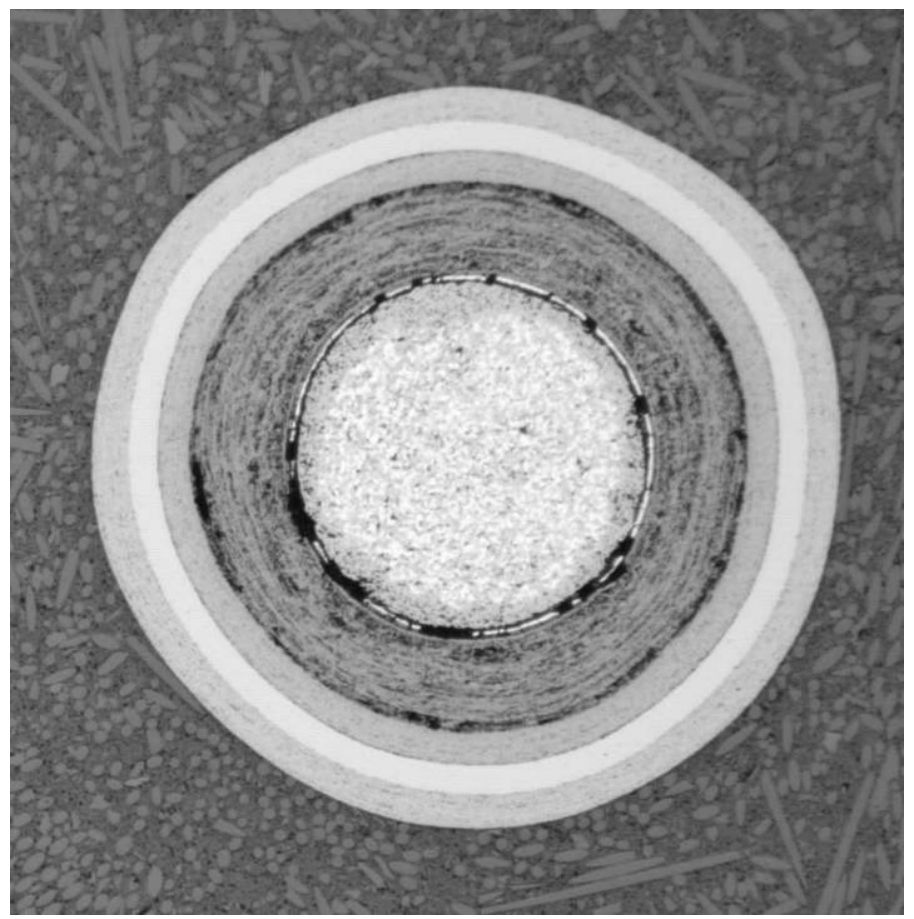

Figure 1. Cross section of a TRISO-coated nuclear fuel particle showing coating layers. 
TRISO coatings are applied in a graphite vessel at temperatures well in excess of service temperatures for many materials and instruments $\left(1300^{\circ} \mathrm{C}-1500^{\circ} \mathrm{C}\right)$ and with gas environments that include dense particulate clouds, corrosive gases, and hydrogen concentrations over $45 \%$ by volume (Table 1). The severe coating temperatures exceed the service temperature of many metals and materials commonly used for probes. Stringent chemical purity requirements for the coated particles further limit the choice materials because contamination from probe abrasion can compromise in-pile particle performance by leading to an increased probability of coating failures [1].

Table 1. Approximate coating process conditions

\begin{tabular}{|c|c|c|c|}
\hline Coating & Inlet gases & Exhaust gases & $\mathbf{T}_{\text {coating }}\left({ }^{\circ} \mathbf{C}\right)$ \\
\hline Buffer & $\begin{array}{l}60 \% \mathrm{C}_{2} \mathrm{H}_{2} \\
40 \% \mathrm{Ar}\end{array}$ & $\begin{array}{l}60 \% \mathrm{H}_{2} \\
40 \% \mathrm{Ar}\end{array}$ & 1430 \\
\hline $\begin{array}{c}\text { Pyrocarbo } \\
\mathrm{n}\end{array}$ & $\begin{array}{l}70 \% \mathrm{Ar} \\
16.2 \% \mathrm{C}_{2} \mathrm{H}_{2} \\
13.8 \% \mathrm{C}_{3} \mathrm{H}_{6}\end{array}$ & $\begin{array}{l}55 \% \mathrm{Ar} \\
45 \% \mathrm{H}_{2}\end{array}$ & 1285 \\
\hline $\begin{array}{l}\text { Silicon } \\
\text { Carbide }\end{array}$ & $\begin{array}{l}98.5 \% \mathrm{H}_{2} \\
1.5 \% \mathrm{CH}_{3} \mathrm{SiCl}_{3}\end{array}$ & $\begin{array}{l}95.6 \% \mathrm{H}_{2} \\
4.4 \% \mathrm{HCl}\end{array}$ & 1475 \\
\hline
\end{tabular}

Additionally, the need to assure isolation of the hydrogen atmosphere from oxygen sources and control of radioactive and fissile materials preclude the insertion of many kinds of probes and instruments into the coater vessel during operation. Furthermore, the presence of probes in the bed could result in damage to delicate TRISO coating layers as they are forming on the particles; resulting in an increased product reject fraction and increased probability of coating failures during fuel irradiation.

The coatings are applied by thermally decomposing reactant gases to form either a carbon or silicon carbide layer on the particles. The gases must be kept relatively cool as they pass through the distributor nozzle to prevent premature decomposition and excessive distributor fouling. This is achieved by using unheated coating gases and diverting some of the diluent gas (argon or hydrogen) around the nozzle exterior to affect some cooling of the nozzle flange in contact with the coating vessel.

The gases heat up rapidly as they pass through the distributor nozzle and enter the bed, resulting in nearly explosive expansion of the gases. The thermal expansion is augmented by an increase in the total moles of gas, due to decomposition of the coating gases, for all coatings but the buffer deposition.

Correlations developed and used in industry to calculate minimum gas velocities to fluidize or spout a particle bed do not necessarily apply to the high temperature coating process with the rapid gas expansion at the inlet. The uncertainty regarding the adequacy of the gas flows to properly spout the fuel particles in the coater vessel led to an effort to monitor the pressure fluctuations that have been observed at the gas inlet.

\section{METHOD}

A compact, Kobold high-precision pressure transducer (0 - 10 psig range) was installed on the gas inlet line to the 6inch coater to monitor pressure fluctuations emanating from the fuel bed as a result of bed and gas "bubble" movement. The length of the pressure line was kept as short as reasonably possible to keep signal attenuation and smoothing minimal. The instrument tubing was purged with a slow flow of argon ( $\sim 100 \mathrm{cc} / \mathrm{min})$ to keep the line clear and free of hydrogen and coating gases. A Rosemont differential pressure transmitter was also installed between the gas inlet and the coater exhaust to monitor changes in the differential pressure across the gas distributor and bed. The high-pressure line is in common with the high-precision transducer while the low-pressure line is dedicated to the transmitter.

These instruments were added to provide a glimpse into the dynamics of coater bed during the coating process, to provide data that could be used to help ascertain the adequacy of fluidization, and help identify the dominant fluidization regimes.

Pressure data were collected, using LabView 8.2 software, at prescribed intervals during the deposition of each coating layer; specifically, one minute into the deposition, at the midpoint, and one minute prior to ending the each layer deposition. Changes in the pressure signal structure can be observed as a result of changes in the particle properties (average density, diameter, aspect ratio, etc.). Data were collected for $6-8$ seconds intervals at $400 \mathrm{~Hz}$ and subsequently filtered. The high-precision pressure fluctuation signal was band-pass filtered between $0.25 \mathrm{~Hz}$ and $150 \mathrm{~Hz}$ to preclude aliasing and to center the data at an average of zero to facilitate comparisons of one signal to another. Differential pressure data were low-pass filtered at $3 \mathrm{~Hz}$ to smooth fluctuations and provide the mean differential pressure across the gas distributor and the particle bed. Raw (unfiltered) pressure data were used to determine total system back pressure, as measured from the gas inlet.

Filtered pressure data were analyzed using power spectral densities (PSD), short-term Fourier transforms (STFT), and calculating Hurst exponents (rescaled range analysis). Peak amplitude and frequency data were extracted from the PSDs, and the regressed slopes (Hurst exponents) and standard deviations were extracted from ranges on the Pox plots [2].

The Hurst exponents are estimated by the slopes of the line on a rescaled range plot (Pox diagram), as shown in Figure 2, computed for given ranges along the abscissa (Table 2). Slopes having values near 1.0 indicate a persistent trend in the sample data (i.e., successive data points predict the trend of subsequent points), slopes near 0.5 indicate a randomness to the data, and slopes near 0.0 indicate an antipersistent trend. The periodic nature of the pressure fluctuations is manifested, in this case, 
by the undulating segment of the rescaled range plot beyond a lag of 70 observations.

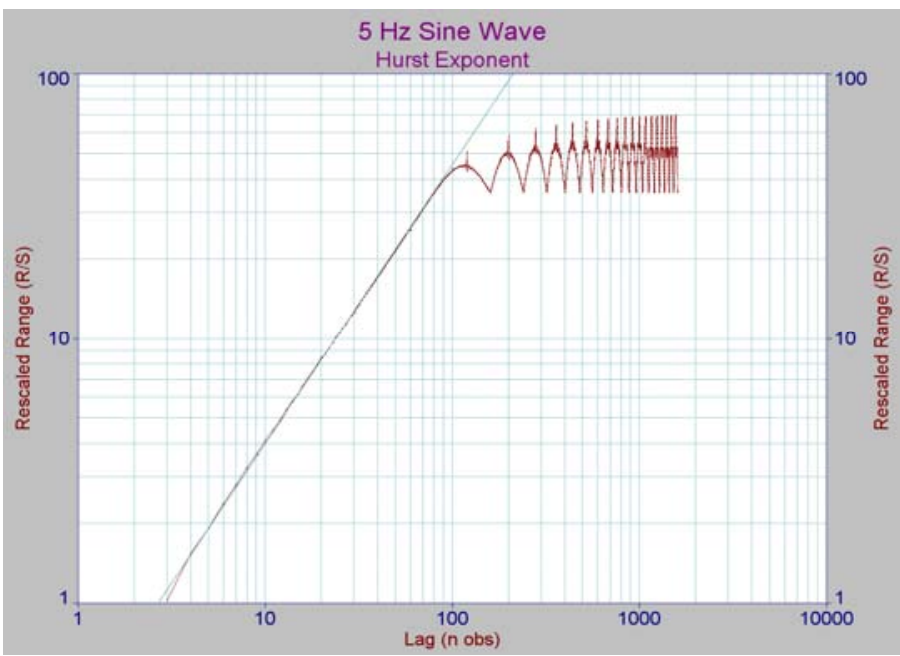

Figure 2. Pox diagram of a $5-\mathrm{Hz}$ sine wave sampled at $400 \mathrm{~Hz}$.

The selected ranges in Table 2 were chosen from natural breaks in the average slope observed over several Pox diagrams obtained during the particle coating tests. The break points fit well with most of the layers, which exhibit changes in slope, but are less than ideal for the buffer layer that would be better described by a narrower range for the $\mathrm{H}_{1 \mathrm{~b}}$ exponent. For this study, the ranges on the Pox diagram for the Hurst exponent estimates were not customized for each respective layer.

Table 2. Ranges from which Hurst exponents were estimated.

\begin{tabular}{|l|l|}
\hline Hurst exponent & Lag range along abscissa \\
\hline $\mathrm{H}_{1 \mathrm{a}} \pm \sigma_{1 \mathrm{a}}$ & $3 \leq \mathrm{n}$ obs $\leq 20$ \\
\hline $\mathrm{H}_{1 \mathrm{~b}} \pm \sigma_{1 \mathrm{~b}}$ & $20 \leq \mathrm{n}$ obs $\leq 70$ \\
\hline $\mathrm{H}_{2} \pm \sigma_{2}$ & $120 \leq \mathrm{n}$ obs $\leq 600$ \\
\hline $\mathrm{H}_{3} \pm \sigma_{3}$ & $600 \leq \mathrm{n}$ obs $\leq 1600$ \\
\hline
\end{tabular}

The Hurst exponents for a $5-\mathrm{Hz}$ sine wave, estimated from the slopes of the curve on the Pox diagram in Figure 2, are given below and clearly show persistency for low lag values and antipersistency for higher lag values.

$$
\begin{array}{ll}
\mathrm{H}_{1 \mathrm{a}}: & 1.0536 \pm 0.0058 \\
\mathrm{H}_{1 \mathrm{~b}}: & 1.0539 \pm 0.0027 \\
\mathrm{H}_{2}: & 0.1174 \pm 0.0143 \\
\mathrm{H}_{3}: & 0.0689 \pm 0.0209
\end{array}
$$

\section{DISCUSSION AND RESULTS}

Many methods commonly used to estimate the minimum fluidizing velocity for a bed of particles incorporate the
Archimedes number (Equation 1) into the calculation of the particle Reynolds number at incipient fluidization $[3,4,5]$. Similarly, correlations for estimating the minimum spouting velocity of a spouted particle bed are proportional to the particle diameter and particle density as shown in Equation 2 [6].

$$
\begin{aligned}
& A r=\frac{d_{p}^{3} \rho_{g}\left(\rho_{p}-\rho_{g}\right) g}{\mu^{2}} \\
& U_{m s}=f\left(d_{p}, \rho_{p}, \rho_{g}, D_{c}, D_{o r}, H_{b e d}\right)
\end{aligned}
$$

The first two attributes listed in Equation 2 are the mean particle diameter and absolute particle density. The other attributes are the mean gas density, column diameter, gas inlet orifice diameter, and bed height. It is clear that particle attributes have significant impacts on particle fluidization. Other parameters that have been identified as contributors when scaling a spouted bed include the particle sphericity, and material properties such as the angle of internal friction [7] and the coefficient of restitution [8]. It is reasonable to assume that pressure fluctuations emanating from the bed as a result of bed and bubble movement would be influenced by particle properties. If so, then one could hypothesize that the structure of the pressure fluctuation signal may reveal information about the collective particle attributes.

Table 3 gives the coating layer and particle properties, assuming a spherical particle, along with target coating layer thicknesses and representative layer densities. The table also shows the volume expansion relative to the initial charge of kernels. Actual layer and average particle properties will vary depending on particle sphericity and mean coating layer thickness and density.

Table 3. Particle and layer properties

\begin{tabular}{|lccccc|}
\hline & $\begin{array}{c}\text { Layer } \\
\text { thickness } \\
\Theta_{\mathrm{L}}(\mu \mathrm{m})\end{array}$ & $\begin{array}{c}\text { Layer } \\
\text { density } \\
\rho_{\mathrm{L}} \\
(\mathrm{g} / \mathrm{cc})\end{array}$ & $\begin{array}{c}\text { Particle } \\
\text { diameter } \\
\mathrm{d}_{\mathrm{p}}(\mu \mathrm{m})\end{array}$ & $\begin{array}{c}\text { Particle } \\
\text { density } \\
\rho_{\mathrm{p}} \\
(\mathrm{g} / \mathrm{cc})\end{array}$ & $\begin{array}{c}\text { Vol. } \\
\text { ratio } \\
\mathrm{V} / \mathrm{V}_{\mathrm{K}}\end{array}$ \\
\hline Kernel & --- & --- & 429 & 10.71 & 1.00 \\
\hline Buffer & 100 & 1.05 & 629 & 4.11 & 3.15 \\
\hline $\mathrm{IPyC}$ & 40 & 1.89 & 709 & 3.44 & 4.51 \\
\hline $\mathrm{SiC}$ & 35 & 3.20 & 779 & 3.38 & 5.99 \\
\hline OPyC & 40 & 1.89 & 859 & 3.00 & 8.03 \\
\hline
\end{tabular}




\section{Buffer Layer}

Deposition of the $100 \mu \mathrm{m}$ carbon buffer layer on the fuel kernels results in a $60 \%$ decrease in particle density and more than a $300 \%$ increase in particle/bed volume. The initial static bed depth of the bare kernels in the coater is estimated to be $3.9 \mathrm{~cm}$, which is too shallow to form a well-developed spout or particle bed circulation pattern until most of the buffer has been applied. Evidence of a shift in bed fluidization during buffer deposition is seen in the power spectral density (PSD) and short-term Fourier transform plots (STFT), shown in Figures 3 and $4 \mathrm{a}-\mathrm{c}$.

\section{Buffer PSD}

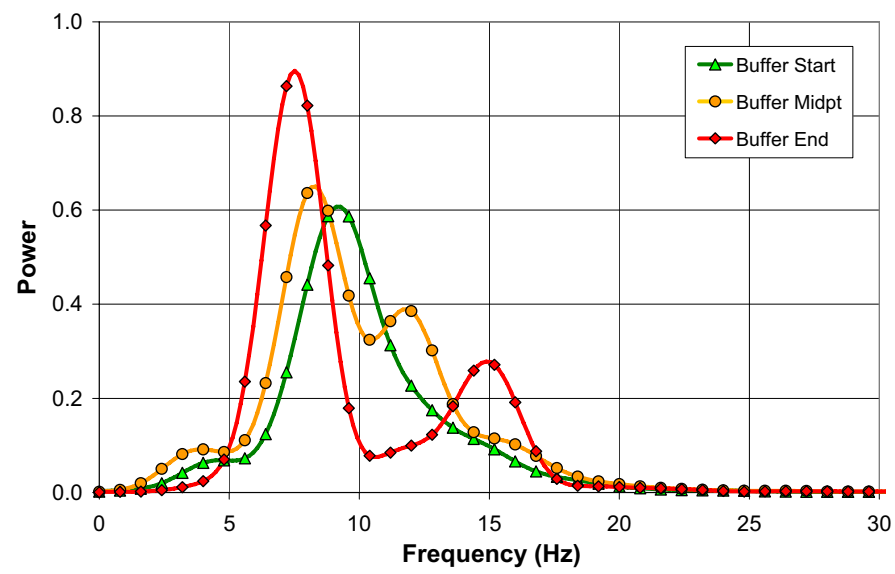

Figure 3. Representative PSD of the buffer deposition.

The starting PSD plot shows a relatively broad, monomodal power distribution and the STFT for the same sample period shows a weak structure over time. The midpoint sample exhibits a bimodal PSD and a disordered STFT structure. It is interesting to note that the buffer sample taken at the end of the deposition period exhibits a bimodal distribution for the PSD and a strongly structured STFT, as evidenced by the columnar contour plot. The presence of a bimodal distribution on the PSD and strong structure on the STFT plot is considered to be indicative of a desirable fluidization regime for the coater used to generate this data.

The change in the average particle properties and bed mass produces a progressive shift in the PSD peak power to lower frequencies. Plots of the buffered particle diameter vs. the change in peak-power frequency and the ratio of the ending and starting frequencies are given in Figures 5 and 6 . The buffered particle diameters were calculated based on average kernel diameters and measured buffer thicknesses obtained from sectioned particles. These figures confirm that the shift in peak- power frequencies is influenced by the particle properties. The use of the frequency difference or a frequency ratio is insensitive to the initial kernel charge masses of $1.3 \mathrm{~kg}$ or $1.5 \mathrm{~kg}$.

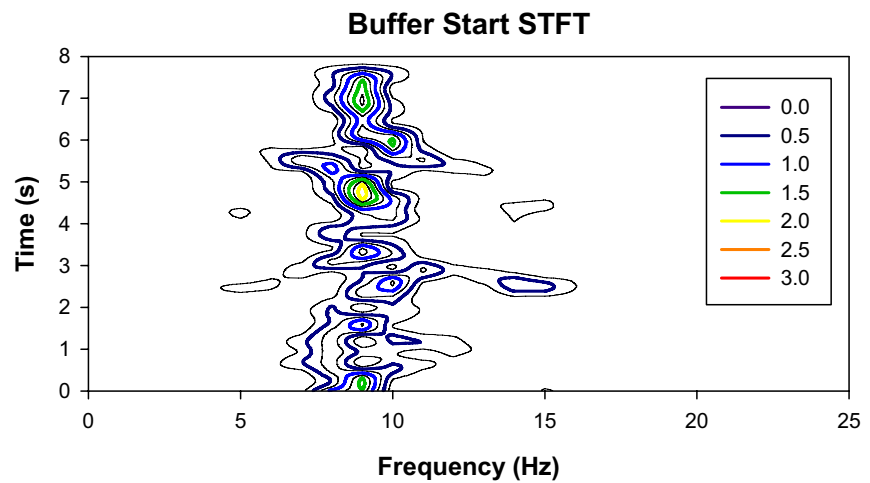

Figure 4a. Representative STFT plot at the start of buffer deposition.

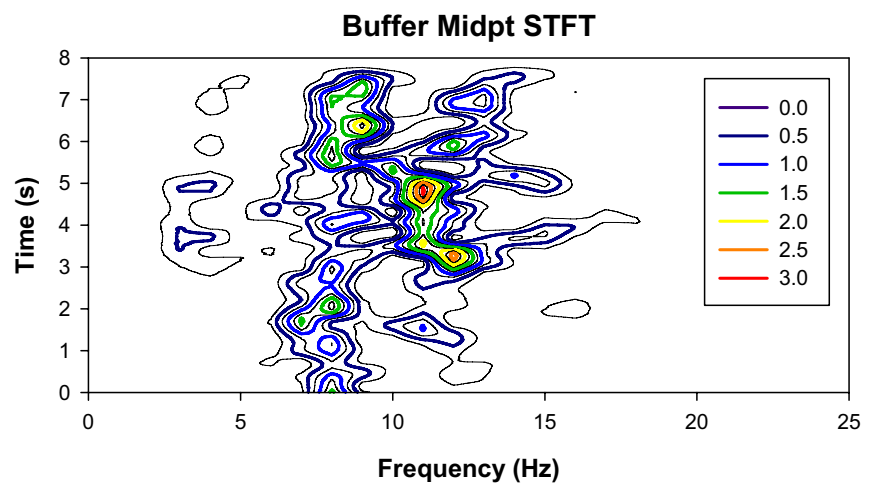

Figure 4b. Representative STFT plot at the midpoint of buffer deposition.

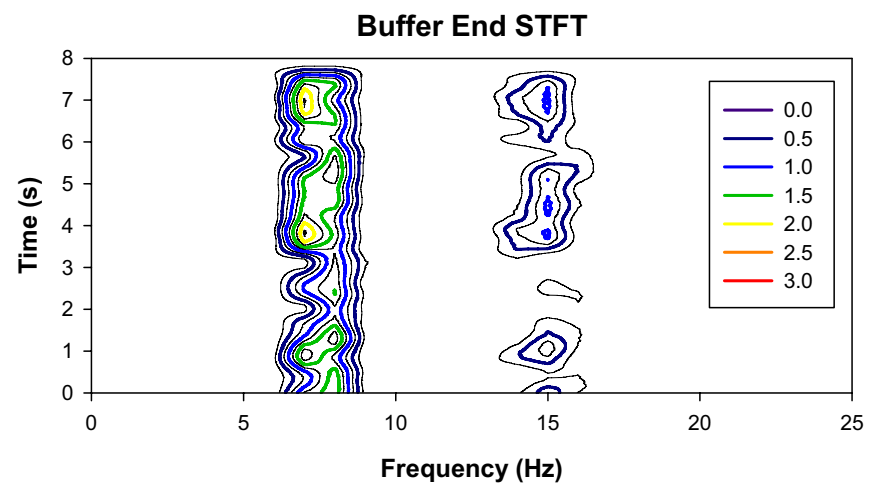

Figure 4c. Representative STFT plot at the end of buffer deposition.

However, the poorly structured fluidization at the start of the buffer deposition and variability in the timing of manual pressure fluctuation data collection have introduced sufficient uncertainty in the peak-power frequency determinations to preclude a precise estimation of the buffered particle diameter or the thickness of the buffer layer. 


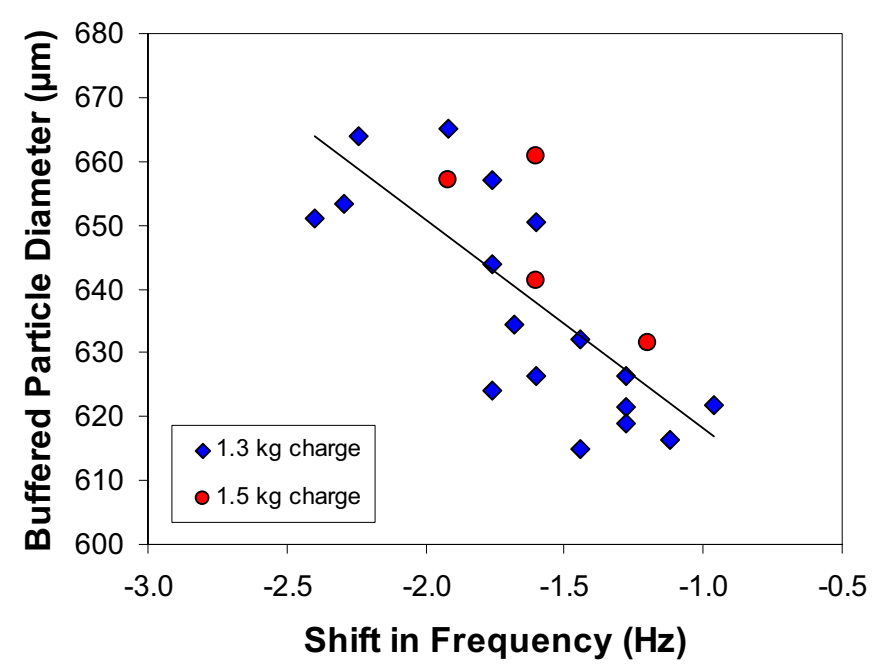

Figure 5. Plot of the the buffered particle diameter vs. a shift in the PSD peak-power frequencies $\left(\omega_{1 \text {, end }}-\omega_{1 \text {, start }}\right)$.

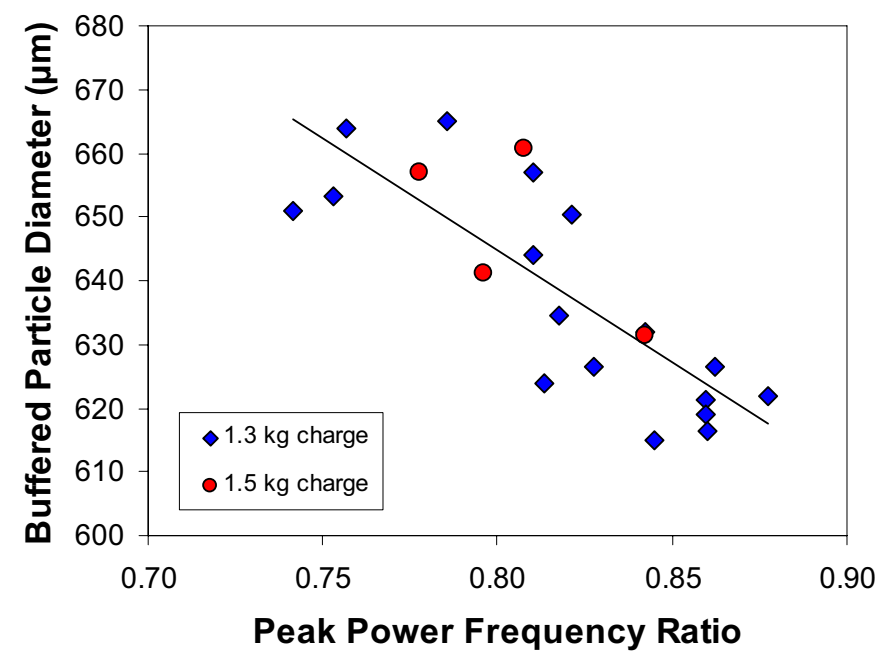

Figure 6. Plot of the buffered particle diameter vs. the ratio of PSD peak-power frequencies $\left(\omega_{1, \text { end }} / \omega_{1 \text {, start }}\right)$.

Buffer density determinations require that particle samples be drawn from the coater vessel after the buffer deposition and prior to subsequent coatings. Consequently, only limited data are available. When samples are available for buffer density determinations, the procedure uses pressured mercury to obtain the "envelope" density of the coated kernel and the buffer density is calculated by extracting the kernel contributions to the mass and diameter. Variability in the kernel diameter and the delicate nature of the buffer (easily curshed) result in substantial uncertainty and scatter in the buffer density determinations.

Figure 7 shows a possible relationship between the density of the buffer coating and the change in the average backpressure detected at the gas inlet (including the sum of the gas distributor, bed, and soot filter differential pressures). Buffer density data are sparse because of limited capacity to obtain "hot" buffer samples. The density analysis is imprecise because buffer density is inferred from analysis of the buffercoated particles via a mercury pycnometry technique. Taken collectively, the data seem to show a trend correlating pressure buildup in the coater with density of the buffer coating, but taken separately by charge mass, the data appear to be less convincing

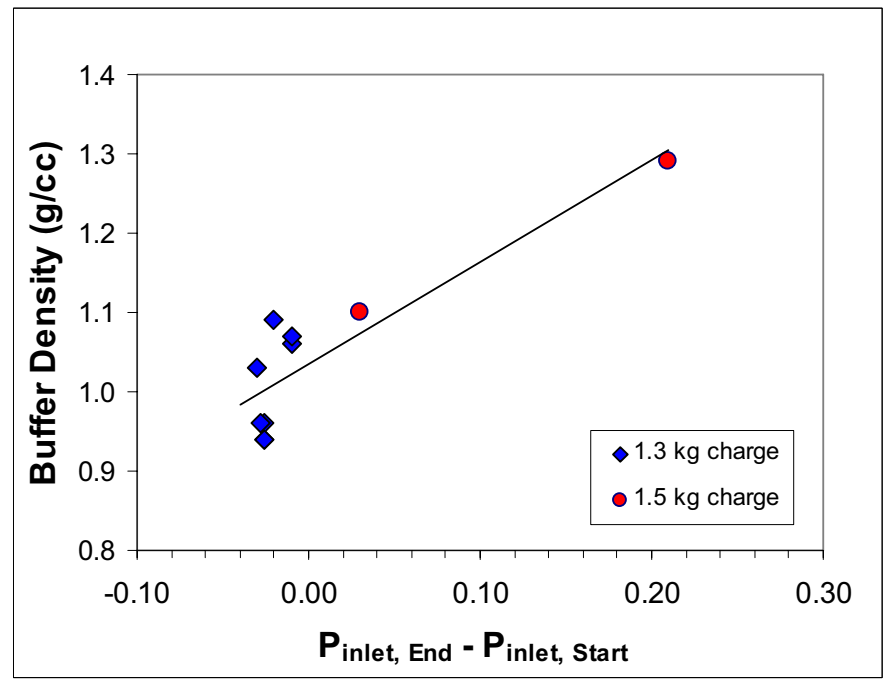

Figure 7. Plot of buffer density correlated with the change in coater gas inlet backpressure.

Figure 8 shows a possible relationship between the calculated buffer-coated particle density and the ratio of the dominant peak frequencies from the PSD plots $\left(\omega_{\text {end }} / \omega_{\text {start }}\right)$. As previously observed with the buffered particle diameters, the particle density appears to correlate well the ratio of ending to starting characteristic frequencies and to be independent of the charge mass within the range of charge masses tested in this study.

The apparent precision in the buffer density predictions shown in Figures 7 and 8 may be artifacts of limited data. As more data come available, more extensive data scatter will likely be manifested in the plots. 


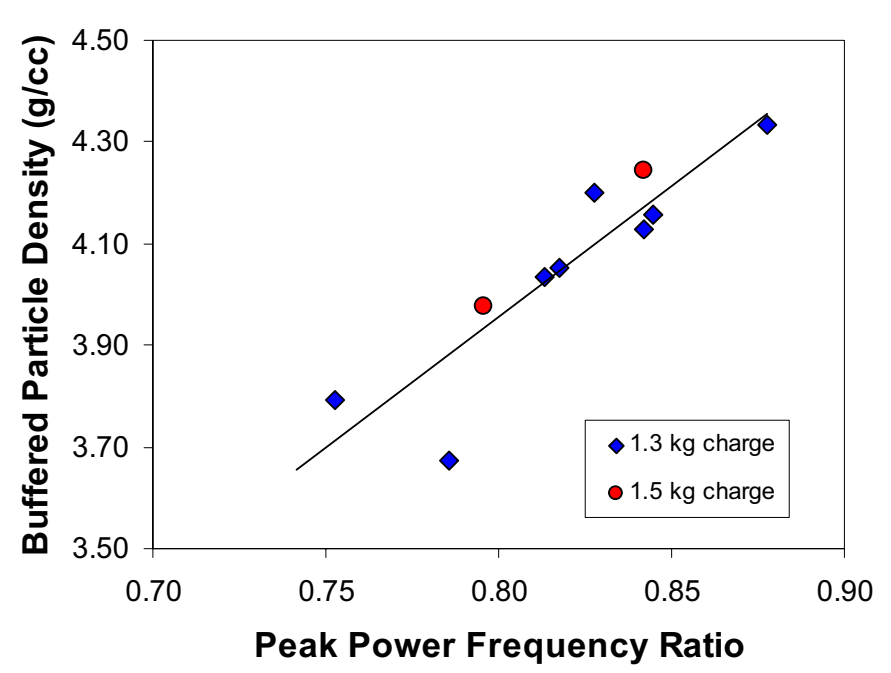

Figure 8. Plot of buffer density vs. the ratio of PSD peakpower frequencies $\left(\omega_{1, \text { end }} / \omega_{1, \text { start }}\right)$.

\section{Inner Pyrocarbon (IPyC) Layer}

Early coating process development efforts focused on the use of spherical zirconia beads as a surrogate kernel to avoid the expense of forming natural uranium oxide - uranium carbide spheres (a.k.a. natural uranium oxycarbide - NUCO).

The density of yttria-stabilized zirconia is approximately $6.0 \mathrm{~g} / \mathrm{cc}$ and the density of NUCO kernels is near $10.9 \mathrm{~g} / \mathrm{cc}$. However, a buffer coated NUCO particle has a density near 4.2 $\mathrm{g} / \mathrm{cc}$, which makes it possible to select a zirconia kernel with a mean diameter and density similar to that of a NUCO kernel with about half of the buffer layer applied. All of the recognized scaling relations were closely matched $[7,8]$, which are thought to provide hydrodynamic similitude.

In spite of this effort to achieve similitude with the coated particles, differences in the thermal diffusivities and/or rotational inertia of the partially-coated particles may have led to differences in the apparent relationships between the structure of the pressure fluctuations and particle properties. An example of this phenomenon is the linear regression of the inner pyrocarbon (IPyC) diattenuation and the standard deviation of the Hurst exponent estimated with a lag of $600 \leq n$ obs $\leq 1600$ (Figure 9). The regression fit factor $\left(\mathrm{R}^{2}\right)$ is similar for the coated surrogate and NUCO coated particles, but the slopes of the regressions have opposite signs.

An effort has been made to use relationships that are consistent in the slope magnitude and sign throughout the study. Many promising relationships have been discounted because of inconsistencies between correlations for the surrogate and the NUCO particles. It is not known whether the differences arise because the thermal properties of the kernels influencing the rate of heat exchange with the incoming coating gases, whether the differences in particle rotational inertia and particle angular velocities influence particle-particle interactions, or if the apparent relationships are falsely indicated.

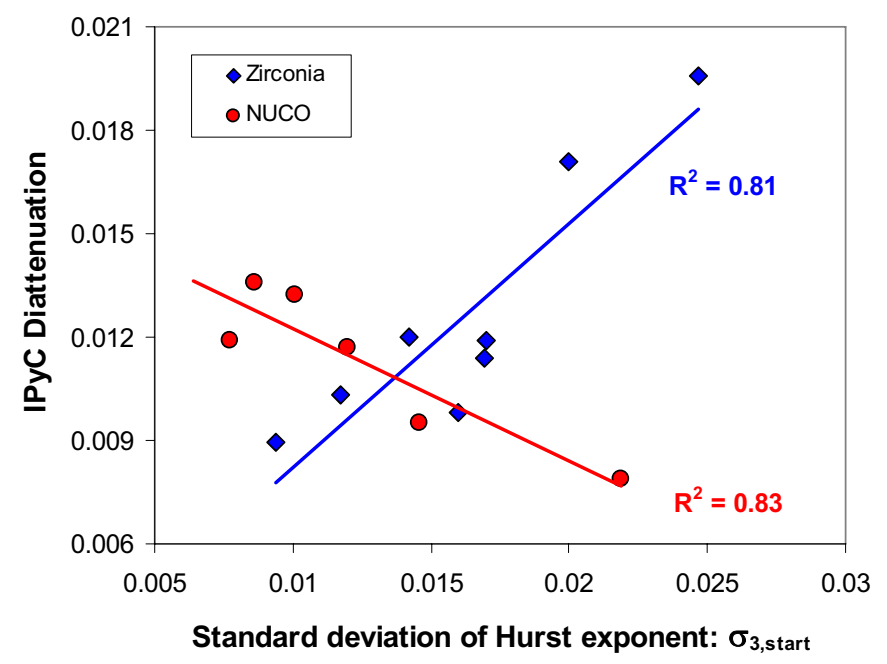

Figure 9. Example of apparent disagreement in relationships.

Contrary to expectations, no consistent relationships were discovered for estimating the diameter and density for the IPyC coated particles or the IPyC thickness.

There are apparent correlations with coating attributes that are less intuitive, but may be impacted by the same gas and particle properties that affect the fluidization. One of these is the anisotropy (diattenuation) of the pyrocarbon layers (Figure 10).

Anisotropy is a measure of the degree of persistent structure or order in the pyrolytic carbon crystallites and is used as in predictor of coating layer performance during irradiation. A proven correlation between the pyrocarbon anisotropy and pressure signals could be used to screen product, thereby reducing the sampling frequency and possibly signaling unexpected process changes in the pyrocarbon deposition; thereby avoiding subsequent production runs yielding product that does not meet specifications.

The inner pyrocarbon (IPyC) anisotropy in Figure 10 was predicted via a quadravariate linear regression of the Hurst exponents and the change in coater inlet backpressure (Equation 3). The particles with a zirconia kernel were regressed independently from those with a NUCO kernel. Although the coefficients may differ in value, their signs are consistent for each of the terms.

$N_{I P y C}=N_{o}+a \cdot H_{1 a, \text { Mid }}+b \cdot \sigma_{3, \text { Mid }}+c \cdot H_{2, \text { End }}+d \cdot\left(P_{\text {inlet }, \text { End }}-P_{\text {inlet }, \text { Start }}\right)$

Eq. 3 


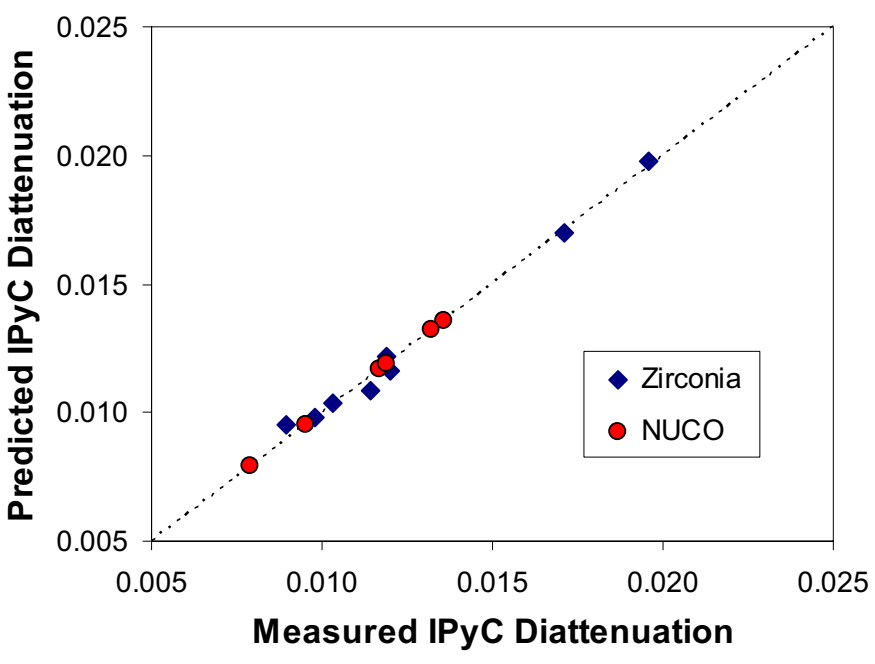

Figure 10. Predicted vs. measured IPyC diattenuation.

It is expected that the amount of data scatter in Figure 10 will increase as more data become available and are included in the regression.

The IPyC layer density (Figure 11) was fitted to a parabolic equation of a Hurst exponent mid-way through the coating deposition $\left(\mathrm{H}_{1 \mathrm{amidpoint}}\right)$ and the extreme spread of pressures sampled at the end of the IPyC deposition (Equation 4). Some uncertainty is introduced by run-to-run variability in the average particle diameters and densities of the underlying buffer-coated particle and the final IPyC-coated particle, which diminishes the precision of the pyrocarbon density estimate.

$\rho_{I P y C}=\rho_{o}+a \cdot P_{E S, \text { End }}+b \cdot P_{E S, \text { End }}^{2}+c \cdot H_{1 a, \text { Mid }}+d \cdot H_{1 a, \text { Mid }} \quad$ Eq. 4

The mass of the bed following IPyC deposition can be calculated from measured and assumed densities, diameters, and thicknesses for the kernel, buffer, and IPyC layers. The uncertainties in the measured and calculated properties reduce the precision of the bed mass prediction. The estimated bed mass seems to correlate with the extreme spread of the pressure fluctuations at the coating midpoint, the standard deviation of the midpoint pressure fluctuations, the amplitude of the minor peak in the power spectral density and the frequency of the major peak on the PSD at the end of the coating (Equations 5). The segregation of data by the initial charge mass is evident in Figure 12 as evidenced by the clustering of the data around different estimated bed masses. No other physical properties of the IPyC-coated particle seem to correlate well and consistently, between the kernel types, with the pressure fluctuation signals.

$$
M_{\text {bed }}=a \cdot P_{E S, \text { Mid }}+b \cdot \sigma_{\text {fluct,Mid }}+c \cdot A_{2, \text { Mid }}+d \cdot \omega_{1, \text { End }} \quad \text { Eq. } 5
$$

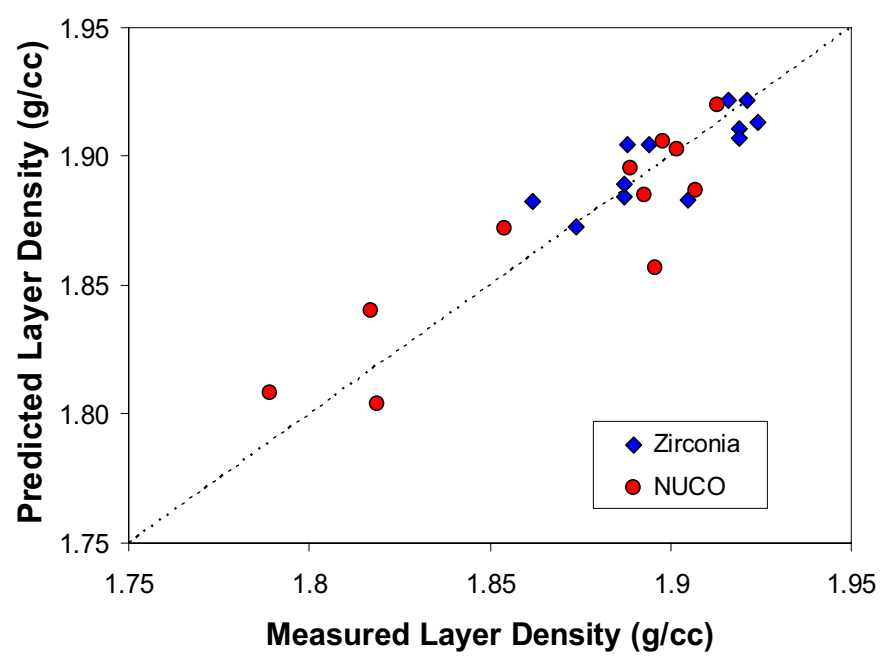

Figure 11. Predicted vs. measured IPyC density.

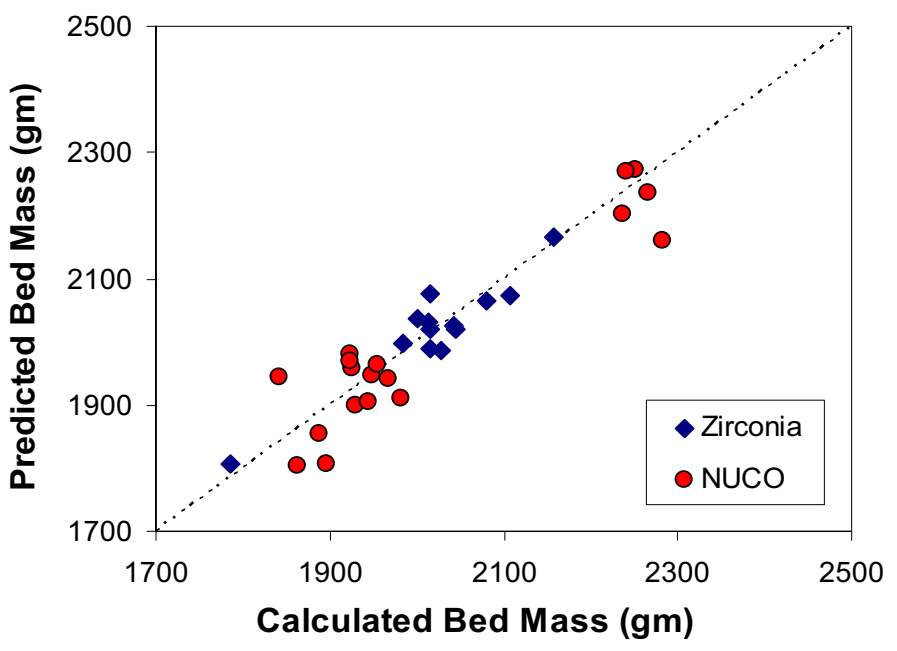

Figure 12. Predicted vs. calculated bed mass after IPyC deposition.

\section{Silicon Carbide Layer}

An important attribute that is measured on the silicon carbide ( $\mathrm{SiC}$ ) coating layer is the aspect ratio, which is an indicator of particle sphericity. This ratio is computed from the radial maxima and minima of a particle cross section measured from the geometric center of the particle. A high aspect ratio $(>1.06)$ leads to excessive stress accumulation in the $\mathrm{SiC}$ layer and could lead to in-pile $\mathrm{SiC}$ failures and fission product releases from the particle. No data are available for the $\mathrm{SiC}$ aspect ratio of the coated zirconia surrogate particles, so the pressure data correlated with the aspect ratio for coated NUCO particles cannot be refereed for consistency. Nonetheless, the data presented in Figure 13 hold promises for future prediction 
of the $\mathrm{SiC}$ aspect ratio when correlated with the standard deviations and extreme spread of the pressure fluctuations and the frequency of the dominant peak on the PSD (Equation 6). The prediction of $\mathrm{SiC}$ aspect ratio is not independent of whether the fluidizing gas is hydrogen or a hydrogen-argon blend in spite of deliberate efforts to achieve similar fluidization by adjusting the total gas flow to the coating reactor. It is thought that the presence of argon in the fluidizing gas, the somewhat lower deposition temperatures, and the lower superficial gas velocities may influence the nature of $\mathrm{SiC}$ fouling on the coater wall and therefore change the particlewall interaction. Furthermore, the accretions that collect on the gas injection nozzle restrict the gas ports during the argon variant runs than otherwise. This influences the orifice gas velocity and the total backpressure, which is reflected in changes to some regressed coefficients. Consequently, the coefficients for the equation were regressed separately for the two cases.

$A R_{S i C}=1+a \cdot \sigma_{f l u c t, M i d}+b \cdot P_{E S, E n d}+c \cdot \sigma_{f l u c t, \text { End }}+d \cdot \omega_{1, \text { Mid }} \quad$ Eq. 6

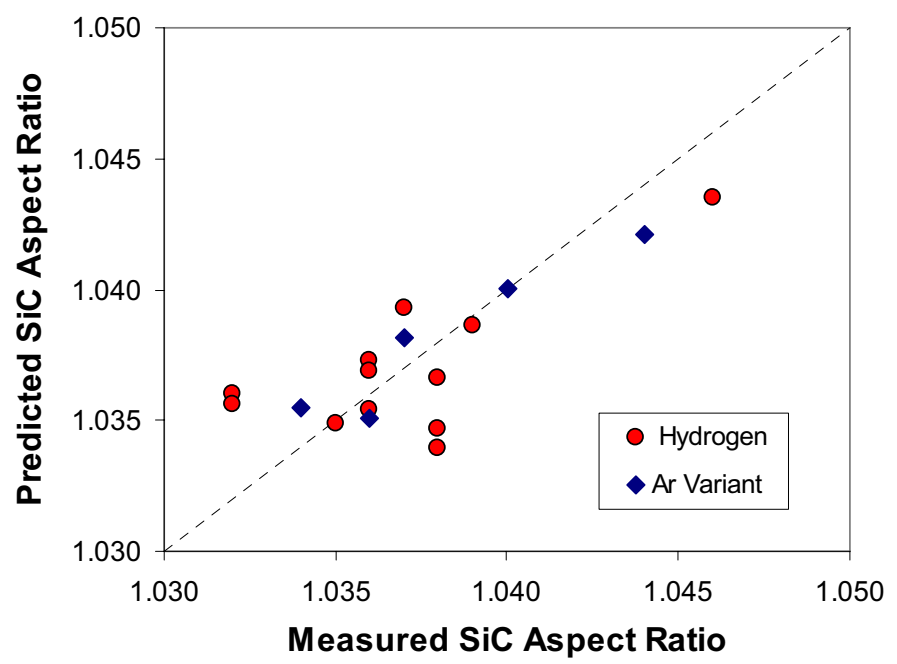

Figure 13. Predicted vs. measured SiC aspect ratio.

Although correlations were found for additional particle properties (e.g., particle density, particle diameter, bed mass, etc.), none were consistent between the coated zirconia surrogate and NUCO kernels; differing in the signs on the regressed coefficients and statistical fit.

\section{Outer Pyrocarbon Layer}

Many of the OPyC particle properties are calculated based on measured values for the previous layer thicknesses and densities. Errors propagated from one layer to subsequent layers make it difficult to reliably quantify relationships between pressure signals and particle properties. The anisotropy, or rather the diattenuation of the outer pyrocarbon $(\mathrm{OPyC})$ layer is not dependent on measured substrate values. The variables that correlate well with the $\mathrm{OPyC}$ diattenuation are the final coater inlet differential pressure and the standard deviation of fourth Hurst exponent at the start of the OPyC deposition, and change in gas inlet back pressure over the course of the deposition (Equation 7, Figure 14).

$$
N_{O P y C}=N_{0}+a \cdot D P_{\text {inlet, Start }}+b \cdot \sigma_{3, \text { Start }}+c \cdot\left(P_{\text {inlet }, \text { End }}-P_{\text {inlet }, \text { Start }}\right) \text { Eq. } 7
$$

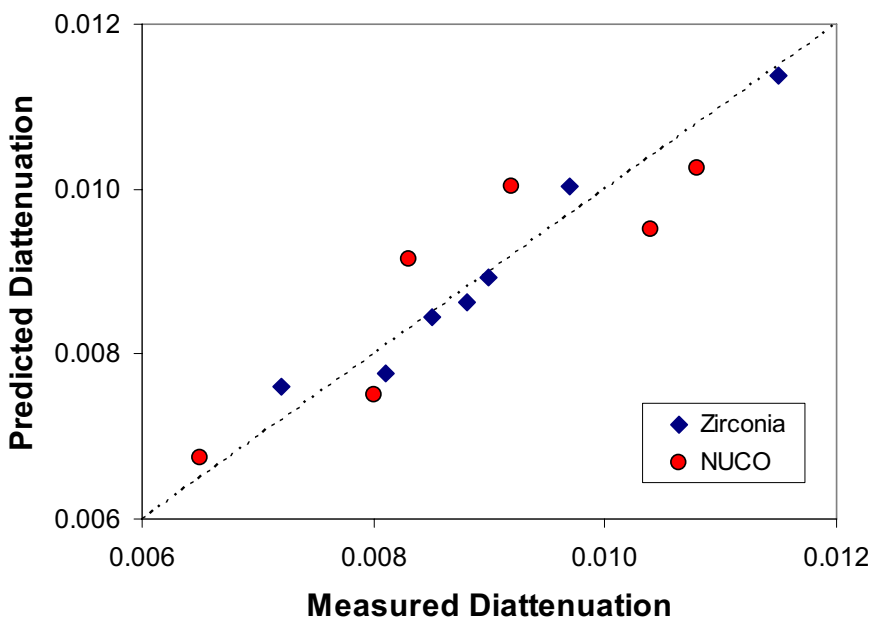

Figure 14. Predicted vs. measured OPyC diattenuation.

The regression for the OPyC diattenuation of coated NUCO particles appears to have a very good fit. This is partially due to the limited data. As additional data become available, the scatter in the plot in Figure 14 is expected to increase.

Considerable data do exist for the OPyC layer density, which have been correlated with the standard deviations of three Hurst exponents; two at the start of the coating and one at the end (Equation 8, Figure 15). The OPyC layer density can be measured by the sink-float method after the layer is broken off of several coated particles. With the accessibility of the OPyC layer for analysis, the need to predict the density has less impact on the program than would the ability to predict IPyC properties. Because the density can be measured directly, the prediction of the density is not dependent on the substrate or subject to a propagation of errors.

$$
\rho_{\text {OPyC }}=\rho_{o}+a \cdot \sigma_{2, \text { Start }}+b \cdot \sigma_{3, \text { Start }}+c \cdot \sigma_{3, \text { End }} \quad \text { Eq. } 8
$$




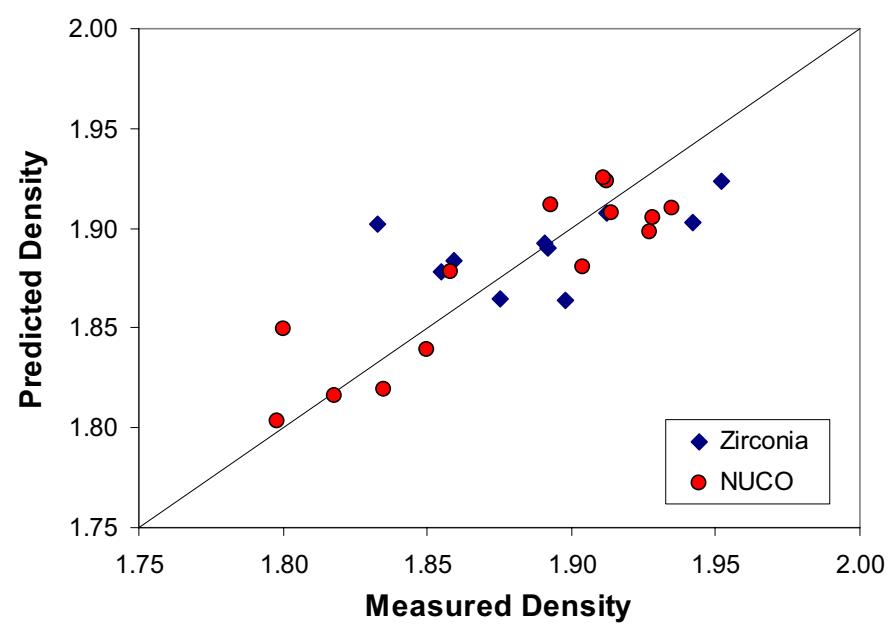

Figure 15. Predicted vs. measured OPyC layer density.

Finally, the aspect ratio of the fully-coated TRISO fuel particle can be estimated based on the standard deviations of Hurst exponents quantified for each of the three sample sets taken during the deposition of the OPyC (Equation 9 and Figure 16). The data show that the amplitude of the pressure fluctuations during OPyC deposition, following the deposition of $\mathrm{SiC}$ in an argon-hydrogen atmosphere, is less than the amplitude following the deposition of $\mathrm{SiC}$ in hydrogen alone. Consequently, the correlations for the aspect ratio were computed for each case. The coating runs using the zirconia surrogate did not include any $\mathrm{SiC}$ depositions in an argonhydrogen atmosphere, but the data were regressed independently from the data from the NUCO runs, because plots of the data for the coated zirconia surrogates and the NUCO appear to be in different populations. The signs of the some coefficients differ between the zirconia and NUCO runs in this case.

$A R_{O P y C}=A R_{o}+a \cdot \sigma_{3, \text { Start }}+b \cdot \sigma_{1 a, \text { End }}+c \cdot \sigma_{1 b, \text { End }}+d \cdot \sigma_{3, \text { End }} \quad$ Eq. 9

One would anticipate that the correlations between similar properties of the IPyC and OPyC coatings would be similar, if not identical sets. For instance, the PSD frequencies, amplitudes, and the Hurst exponents used to predict the IPyC anisotropy ought to be the same set used for the OPyC anisotropies. The same expectation holds for other particle properties. This is not yet the case. It is expected that some insight will be obtained from work being conducted around the world to exploit pressure fluctuation signals in the study of particle bed behavior in fluidized beds.

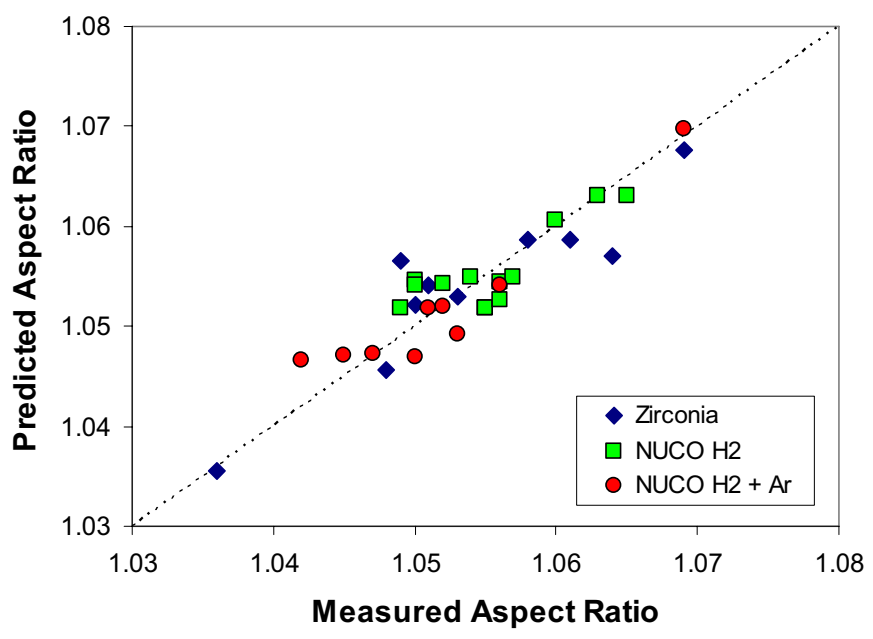

Figure 16. Predicted vs. measured OPyC aspect ratio

\section{CONCLUSIONS}

Pressure fluctuations resulting from the movement of gas through a fluidized particle bed contain some information regarding the particle properties. A variety of techniques have been used to reduce the data to sets that can be correlated to specific properties of the either the particle or the coating layers. The prospect of using pressure fluctuation signals to estimate properties is attractive because predicted values could be available several days before analytical measurements can be completed and reliable correlations could reduce the quantity of samples that would need to be analyzed and reduction of off-specification runs resulting from instrument/controller drift or failures.

Caution is warranted in exploiting this method because:

1. Hurst exponents and their standard deviations have not been mapped to specific physical movements in the bed and the selected ranges were somewhat arbitrary.

2. The connection between pulse frequencies, PSD peaks, and Hurst exponents are described only empirically. A theoretical basis needs to be developed.

3. Due to the highly empirical nature of this study, the results may not be readily exported to systems having different particle or gas properties or a different vessel configuration.

\section{ACKNOWLEDGEMENTS}

Pressure fluctuation data were collected by Babcock \& Wilcox Nuclear Operations Division in Lynchburg, VA during the course of coating tests. 


\section{COPYRIGHT STATEMENT}

This manuscript has been authored by Battelle Energy Alliance, LLC under Contract No. DE-AC07-05ID14517 with the U.S. Department of Energy. The United States Government retains and the publisher acknowledges, by accepting the article for publication, that the United States Government retains a nonexclusive, paid-up, irrevocable, world-wide license to publish or reproduce the published form of this manuscript, or allow others to do so, for United States Government purposes.

\section{NOMENCLATURE}

\begin{tabular}{|c|c|}
\hline $\mathrm{A}_{1}, \mathrm{~A}_{2}$ & $\begin{array}{l}\text { Amplitudes of the first and second peaks on the } \\
\text { power spectral density plots }\end{array}$ \\
\hline $\mathrm{Ar}$ & Archimedes number \\
\hline $\mathrm{AR}_{\mathrm{X}}$ & $\begin{array}{l}\text { Radial aspect ratio (max:min) of the particle } \\
\text { after coating ' } x \text { ' }\end{array}$ \\
\hline $\mathrm{D}_{\mathrm{c}}$ & Diameter of the coater cylinder \\
\hline $\mathrm{D}_{\text {or }}$ & Gas orifice diameter \\
\hline $\mathrm{DP}_{\mathrm{X}}$ & $\begin{array}{l}\text { Differential pressure from gas inlet to exhaust } \\
\text { during coating ' } \mathrm{x} \text { ' }\end{array}$ \\
\hline$d_{p}$ & Mean particle diameter \\
\hline $\mathrm{g}$ & Gravitational constant \\
\hline $\mathrm{H}_{\mathrm{n}}$ & Hurst exponent ' $\mathrm{n}$ ' \\
\hline $\mathrm{H}_{\text {bed }}$ & Height of the particle bed \\
\hline $\mathrm{M}_{\text {bed }}$ & Estimated/calculated bed mass \\
\hline$N_{\text {IPyC }}, N_{\text {OPyC }}$ & Diattenuation of the pyrocarbon layer \\
\hline $\mathrm{P}$ & Gas inlet backpressure \\
\hline $\mathrm{P}_{\mathrm{ES}}$ & $\begin{array}{l}\text { Extreme spread of the pressure fluctuations } \\
\text { during the } 6-8 \text { second sampling }\end{array}$ \\
\hline $\mathrm{U}_{\mathrm{ms}}$ & $\begin{array}{l}\text { Minimum gas velocity required to spout a } \\
\text { particle bed }\end{array}$ \\
\hline $\mathrm{V}, \mathrm{V}_{\mathrm{K}}$ & Particle bed volume ( $\mathrm{K}=$ bare kernels $)$ \\
\hline$\mu$ & Fluidizing gas viscosity \\
\hline$\Theta_{\mathrm{L}}$ & Layer thickness \\
\hline$\rho_{\mathrm{g}}, \rho_{\mathrm{L}}, \rho_{\mathrm{p}}$ & Density of the fluidizing gas, layer, or particle \\
\hline$\sigma_{\text {fluct }}, \sigma_{\mathrm{n}}$ & $\begin{array}{l}\text { Standard deviation of the pressure fluctuations } \\
\text { or the Hurst exponent }\left(\mathrm{H}_{\mathrm{n}}\right)\end{array}$ \\
\hline$\omega_{1}, \omega_{2}$ & $\begin{array}{l}\text { Frequencies of the first and second peaks on the } \\
\text { power spectral density plot }\end{array}$ \\
\hline
\end{tabular}

\section{REFERENCES}

[1] Lessing, P. A., and R. J. Heaps, "Strength of Silicon Carbide Layers of Fuel Particles For HighTemperature Gas-Cooled Reactors," Nuclear Technology, Vol. 108 (1994), 207 - 234.

[2] Xu, Jian, et al., "Characterization of Gas Spouted Beds Using the Rescaled Range Analysis," The Canadian Journal of Chemical Engineering, Volume 82, February 2004, 37- 47.

[3] Geldart, D. (editor), Gas Fluidization Technology, John Wiley \& Sons, 1986.

[4] Davidson, J. F., R. Clift, and D. Harrison, Fluidization $2^{\text {nd }}$ Edition, Academic Press, 1985.

[5] Kunii, D., and O. Levenspiel, Fluidization Engineering $2^{\text {nd }}$ Edition, Butterworth-Heinemann, 1991

[6] Mathur, K. B., and N. Epstein, Spouted Beds, Academic Press, 1974.

[7] He, Y.-L., C. J. Lim, and J. R. Grace, "Scale-up Studies of Spouted Beds," Chem. Eng. Sci., Vol 52 (1997), No. 2, 329-339.

[8] Xu Jian, et al., "Scaling Relationships of Gas-Solid Spouted Beds," 2007 ECI Conference on the $12^{\text {th }}$ International Conference on Fluidization, paper 65. 